\title{
A lethal effect associated with polymorphism of the NOR-bearing chromosomes in rainbow trout (Oncorhynchus mykiss)
}

\author{
Fábio Porto-Foresti ${ }^{1}$, Claudio Oliveira ${ }^{2}$, Eduardo A. Gomes ${ }^{2 *}$, Yara A. Tabata ${ }^{3}$, Marcos G. Rigolino $^{3}$ \\ and Fausto Foresti ${ }^{2}$ \\ ${ }^{1}$ Universidade Estadual Paulista, Faculdade de Ciências, Departamento Ciências Biológicas, \\ Campus de Bauru, Bauru, SP, Brazil. \\ ${ }^{2}$ Universidade Estadual Paulista, Instituto de Biociências, Departamento de Morfologia, \\ Campus de Botucatu, Botucatu, SP, Brazil. \\ ${ }^{3}$ Instituto de Pesca, Núcleo Experimental de Salmonicultura, Campos do Jordão, SP, Brazil.
}

\begin{abstract}
Cytogenetic analysis of a rainbow trout stock showed that the nucleolar organizing regions were located subterminally on the long arm of a submetacentric chromosome pair and occurred as a single chromosomal segment (phenotype N1) or as two chromosomal segments separated by a short euchromatic segment (phenotype N2). Cytogenetic analysis also showed that there were N1N1 and N1N2 individuals but no N2N2 individuals. Analysis of the different includedphenotypes incluted that the population was not in Hardy-Weinberg equilibrium $\left(\chi^{2}=19.333\right.$; $p<0.01$ ), and that a higher frequency of individuals had the N1N2 phenotype. Experimental crosses involving four males (two N1N1 and two N1N2) and four females (one N1N1 and three N1N2) yielded eight broods. There were no significant differences between the expected and observed frequencies of offspring resulting from crosses involving N1N1 x N1N2 individuals. However, significant differences were seen in crosses involving N1N2 $\times$ N1N2 parents because of the a high incidence of N1N2 fishes and the absense of N2N2. The lack of N2N2 individuals in the parental sample and their absence among the offspring of the experimental crosses suggested that this genetic combination may be lethal in rainbow trout. The survival rates of embryonic "eyed egg" and fry stage individuals were not different, indicating that the possible lethal effect may occur during more advanced ontogenetic phases.
\end{abstract}

Key words: NOR polymorphism, paracentric inversion, fish cytogenetics, rainbow trout.

Received: February 10, 2003; Accepted: October 24, 2003.

\section{Introduction}

Salmonid fishes have been extensively studied cytogenetically and numerical and structural variations have been detected in the chromosomes of several species belonging to this family. These alterations probably involve Robertsonian translocations, i.e. the fusion of acrocentric chromosomes or fission of metacentric chromosomes. As a result, there is variation in the diploid chromosome number from $2 n=52$ to $2 n=68$ among trout populations. However, the number of chromosome arms is constant at 104 (Ohno et al., 1965; Ohno, 1974; Thorgaard, 1983; Oliveira et al., 1995).

Studies of the nucleolar organizing regions (NORs) have shown that almost all salmonids have a single pair of

Send correspondence to F. Porto-Foresti, Departamento de Ciêncais Biológicas, Faculdade de Ciências, Universidade Estadual Paulista (UNESP), Campus de Bauru, 17033-360 Bauru, SP, Brazil. E-mail: fpforesti@fc.unesp.br.*Deceased. chromosomes with active NORs (Phillips and Ihssen, 1985; Phillips et al., 1986; Fujiwara et al., 1998). However, other smaller active NORs have also been detected in a number of species (Phillips et al., 1989; Pendás et al., 1993). In the brown trout (Salmo trutta), in addition to larger rDNA clumps detected by the Ag-NOR technique in a specific pair of chromosomes, 16 other smaller regions were identified in the chromosomes using fluorescent in situ hybridization (Pendás et al., 1993). In the rainbow trout, NORs are located subterminally on the short arm of a submetacentric pair of chromosomes (Schmid et al., 1982; Phillips and Ihssen, 1985; Mayr et al., 1986; Ueda and Kobayashi, 1988; Lloyd and Thorgaard, 1988). However, Oliveira et al. (1996), who examined specimens of rainbow trout from the Núcleo Experimental de Salmonicultura (Campos do Jordão, São Paulo, Brazil), observed that NORs were located subterminally on the long arm of a submetacentric pair of chromosomes. In addition, based on staining with silver nitrate these authors identified two 
morphotypes that corresponded to individuals bearing chromosomes with one and two adjacent segments. By studying the synaptonemal complex of these individuals, the difference among NOR-bearing chromosomes was found to result from a paracentric inversion involving this segment. Such a rearrangement has not been previously reported for salmonids. Oliveira et al. (1996) also noted that there were no individuals homozygous for this chromosomal inversion among the trout they examined. This observation suggested that the inversion could be a lethal condition for its carrier.

In the present study, we examined the characteristics and the inheritance patterns of nucleolar organizing regions (NORs) under controlled mating conditions in the rainbow trout stock at the Núcleo Experimental de Salmonicultura.

\section{Material and Methods}

The rainbow trout (Oncorhynchus mykiss) used were obtained through artificial crosses of individuals from stocks maintained at the Núcleo Experimental de Salmonicultura of the Instituto de Pesca, Campos do Jordão, State of São Paulo, Brazil.

Initially the identification and distribution of the NOR phenotpyes were determined by examining a randomly chosen sample of 88 individuals, all of which were marked with magnetic tags and maintained in tanks. Subsequently, eight artificial crosses were done to combine the different NOR phenotypes. These crosses yielded eight $\mathrm{F}_{1}$ groups from which 172 specimens were sampled for cytogenetic analysis.

Cultured lymphocytes (Fenocchio and Bertollo, 1988) or suspensions of kidney cells (Foresti et al., 1993) were used to analyze the chromosomal characteristics of the parental generation and the $\mathrm{F}_{1}$ offspring. Prior to preparation the kidney cell suspension, the trout were injected with a yeast cell suspension to improve the number of metaphase cells (Lozano et al., 1988). The procedure used to identify the NORs was that originally described by Howell and Black (1980). The position of the rDNA genes was confirmed by fluorescent in situ hybridization (FISH) using tilapia $18 \mathrm{~S}$ probes based on the technique described by Porto-Foresti et al. (2002).

The chi-square test (Zar, 1984) was used to compare the observed and expected results.

\section{Results and Discussion}

Cytogenetic analysis of 260 rainbow trout (88 parental specimens and $172 \mathrm{~F}_{1}$ offspring) from the Núcleo Experimental de Salmonicultura showed that the NORs were located subterminally on the long arm of a submetacentric pair of chromosomes, as reported by Oliveira et al. (1996). The analysis also confirmed that the NORs occurred in a single block (N1 phenotype) or as two blocks (N2 phenotype) (Figure 1). The N2 condition resulted from a pericen- tric inversion involving the NOR-bearing chromosomes (Oliveira et al., 1996), as indicated in Figure 1.

The parental stock included 88 specimens (39 males and 49 females). Of the 39 males, 14 were N1N1 (35.9\%) and 25 were N1N2 (64.1\%), with no N2N2. Among the females, 17 were N1N1 (34.7\%), 32 were N1N2 (65.3\%) and there were no N2N2. The chi-square test showed that the phenotype frequencies did not differ significantly between sexes $\left(\chi^{2}=0.064 ; p<0.97\right)$. In contrast, a similar analysis indicated that the population was not in Hardy-Weinberg equilibrium $\left(\chi^{2}=19.333\right.$; $\left.<<0.01\right)$. Differences between the expected and observed proportions were highest in the heterozygous condition (N1N2), indicating that a balanced selection process, i.e. the so-called heterozygotic advantage, could be occurring in this population. The predominance of the heterozygous condition may reflect to a better adaptive capacity of this phenotype compared to individuals with normal homozygous NORs.

Comparison between the observed and expected frequencies of the $\mathrm{F}_{1}$ phenotypes through by specific crosses involving N1N1 breeders indicated that the differences in frequency were not significant $\left(\chi^{2}=0.000 ; p<1.00\right)$. Similar results were observed for comparisons involving the $\mathrm{N} 1 \mathrm{~N} 1$ and N1N2 crosses, regardless of the offspring sex $\left(\chi^{2}=0.1818 ; p<0.9909\right)$. In contrast, comparisons between the observed and expected frequencies of the progenies obtained from crosses between N1N2 individuals revealed highly significant differences in the phenotype proportions $\left(\chi^{2}=17.0000 ; p<0.0002\right)$. The absence of the N2N2

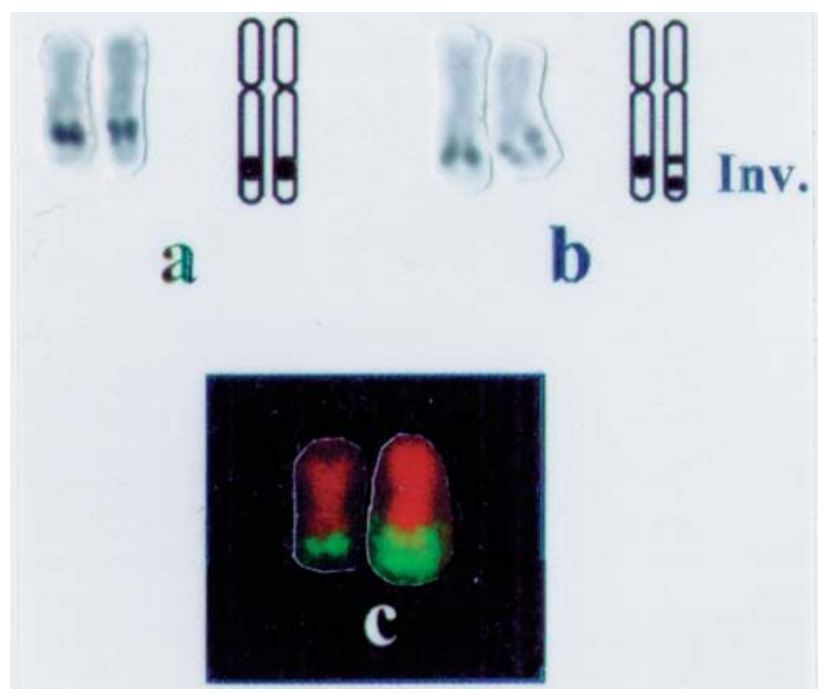

Figure 1 - Photomicrographs and schematic drawings of silver nitratestained NOR-bearing chromosomes of rainbow trout (Oncorhynchus mykiss). a) Chromosome pair and corresponding diagram of a homozygous N1N1 individual, showing the NOR located at an interstitial position on the long arm. b) Chromosome pair of an N1N2 individual heterozygous for a paracentric inversion involving the NOR-bearing segment of the chromosome. The diagram shows the inverted region (Inv). c) Fluorescent in situ hybridization using biotinylated 18S rDNA for chromosomes of rainbow trout. 
phenotypic class accounted for most of the deviation from theoretical values (Figure 2). The high proportion of N1N2 individuals was particularly noteworthy.

The absence of N2N2 homozygotes confirmed the precious suggestion that this could be a lethal condition in this population (Oliveira et al., 1996). The deleterious effects caused by paracentric inversions have been reported for other organisms, with dysfunctions related to protein synthesis being caused by such chromosomic alterations (Swanson et al., 1969). Since mortality is apparently associated with the occurrence of chromosomes homozygous for NOR inversions, cytogenetic markers for this alteration could be extremely important for the commercial breeding of this species. The use of such markers could benefit breeders since the polymorphisms associated with NORs are inherited (Mikelsaar et al., 1977; Markovic et al., 1978; Henderson and Bruere, 1980; Arruda and Monteagudo, 1989; Castro et al. 1998).

Lethal genotypes have also been investigated in other fish especies. Kirpichnikov (1981) described a classic example in Cyprinus carpio, which involved a relationship between the N/S genes and body integument. Lethal homozygosis in this case was similar to that observaded here, with the presence of two N2-type chromosomes presumably resulting in a inviable N2N2 phenotype.

The survival rates of eyed embryos, i.e. individuals with pigmented eyes, and newly hatched larvae were calculated based on the initial number of eggs (Table 1) and did not differ significantly between these two developmental

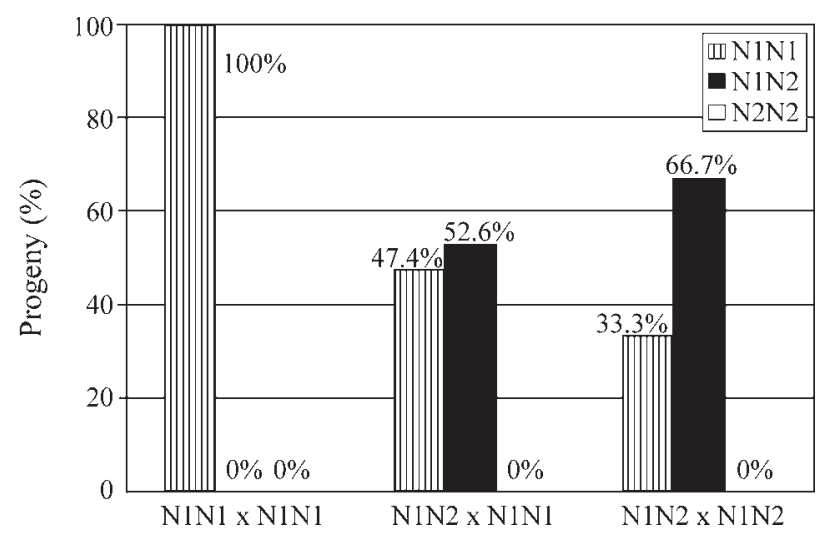

Figure 2 - Progeny of artificial crosses involving different parental combinations of NOR-bearing chromosomes in rainbow trout (Oncorhynchus mykiss). stages. This finding indicated that the deleterious effects associated with the alterations in NOR-bearing chromosomes occurred in more advanced ontogenetic phases.

The present results indicated an association between chromosomal alterations and the occurrence of a detrimental physiological effect at a specific developmental stage. In terms of fish cultivation, this would correspond to a $25 \%$ loss in the expected progeny of specific crosses. On the other hand, the higher proportion of N1N2 individuals in the population suggested that fish with this phenotype may be better adaptated than those with the N1N1 phenotype. Crosses involving N1N1 and N1N2 breeders, from which equal proportions of N1N2 and N1N1 phenotypes are expected in the $\mathrm{F}_{1}$, would yield normal survival rates since no N2N2 offspring and a high number of N1N2 individuals would be produced. On this basis, N1N2 individuals should be identified and their use for reproduction should be limited to N1N1 mates.

More detailed studies on the characterization of this lethal process should be done to improve the management of this stock. Similar research on other local culture stocks and on wild trout populations introduced into Brazil may yield interesting results.

\section{Acknowledgments}

We thank Renato Devidé and Maurício Nagata for technical assistance. This work was supported by CAPES, FAPESP and the Instituto de Pesca - SP.

\section{References}

Arruda MV and Monteagudo LV (1989) Evidence of Mendelian inheritance of the nucleolar organizer regions in the Spanish common rabbit. J Hered 80:85-86.

Castro J, Sánchez L and Matínez P (1998) Analysis of the inheritance of NOR size variants in brown trout (Salmo trutta). J Hered 89:264-266.

Fenocchio AS and Bertollo LAC (1988) A simple method for fresh-water fish lymphocyte culture. Rev Bras Genet 11:847-852.

Foresti F, Oliveira C and Almeida-Toledo LF (1993) A method for chromosome preparations from large specimens of fishes using in vitro short treatment with colchicine. Experientia 49:810-813.

Fujiwara A, Abe S, Yamaha E, Yamazaki F and Yoshida MC (1998) Chromosomal localization and heterochromatin association of ribosomal RNA gene loci and silver-stained nu-

Table 1 - Survival of larvae and eyed embryos in artificial crosses involving breeders of rainbow trout (Oncorhynchus mykiss) with different phenotypes for the NOR-bearing chromosomes.

\begin{tabular}{lccccc}
\hline Crosses & $\begin{array}{c}\text { Initial number of } \\
\text { oocytes }\end{array}$ & $\begin{array}{c}\text { Number of eggs lost up to } \\
\text { the eyed-embryo stage }\end{array}$ & $\begin{array}{c}\text { Eyed embryos } \\
\mathrm{N} / \%\end{array}$ & $\begin{array}{c}\text { Number of eggs lost up Final number of larvae } \\
\text { to the larval stage }\end{array}$ & $\begin{array}{c}\text { N } / \% \\
\text { N1N1 X N1N1 }\end{array}$ \\
361 & 32 & $329 / 91.1$ & 38 & $291 / 80.6$ \\
N1N1 X N1N2 & 1158 & 40 & $1118 / 96.5$ & 161 & $957 / 82.6$ \\
N1N2 X N1N2 & 662 & 34 & $628 / 94.9$ & 54 & $574 / 86.7$ \\
\hline
\end{tabular}


cleolar organizer regions in salmonid fishes. Chromosome Res 6:463-471

Henderson LM and Bruere AN (1980) Nucleolus organizer region location and "ring" chromosomes in the bharal. Experientia 36:176-177.

Howell WM and Black DA (1980) Controlled silver-staining of nucleolus organizer regions with a protective colloidal developer: a 1-step method. Experientia 36:1014-1015.

Kirpichnikov VS (1981) Genetic bases of fish selection. Springer-Verlag, New York, pp 301-319.

Lloyd MA and Thorgaard GH (1988) Restriction endonuclease banding of rainbow trout chromosomes. Chromosoma 96:171-177.

Lozano R, Rejon CR and Rejon MR (1988) A method for increasing the number of mitoses avaliable for cytogenetic analysis in rainbow trout. Stain Tech 66:335-338.

Markovic VD, Worton RG and Berg JM (1978) Evidence for the inheritance of silver-stained nucleolus organizer regions. Hum Genet 41:181-187.

Mayr B, Rab P and Kalat M (1986) Localisation of NORs and counterstain-enhanced fluorescence studies in Salmo gairdneri and Salmo trutta (Pisces, Salmonidae). Theor Appl Genet 71:703-707.

Mikelsaar AV, Schwarzacher HG, Schnedl W and Wagenbichler P (1977) Inheritance of Ag-stainability of nucleolus organizer regions. Investigations in 7 families with trisomy 21 . Hum Genet 38:183-188.

Ohno S (1974) Protochordata, Cyclostomata, and pisces. In: John B (ed) Animal cytogenetics, v. 4, Chordata. GebruderBorntrager, Berlin.

Ohno S, Stenius C, Faisst E and Zenzes MT (1965) Post-zygotic chromosomal rearrangements in rainbow trout (Salmo irideus - Gibbons). Cytogenetics 4:17-29.

Oliveira C, Foresti F, Rigolino MG and Tabata YA (1995) Synaptonemal complex analysis in spermatocytes and oocytes of rainbow trout, Oncorhynchus mykiss (Pisces,
Salmonidae): the process of autosome and sex chromosome synapsis. Chromosome Res 3:182-190.

Oliveira C, Foresti F, Rigolino MG and Tabata YA (1996) Paracentric inversion involving a NOR-bearing chromosome of rainbow trout (Oncorhynchus mykiss): electron microscopy studies of the synaptonemal complex. Caryologia 49:335342.

Pendás AM, Morán P and García-Vazquez E (1993) Multi-chromosomal location of ribosomal RNA genes and heterochromatin association in brown trout. Chromosome Res 1:63-67.

Phillips RB and Ihssen PE (1985) Chromosome banding in salmonid fish: nucleolar organizer regions in Salmo and Salvelinus. Can J Genet Cytol 27:433-440.

Phillips RB, Pleyte KA and Ihssen PE (1989) Patterns of chromosomal nucleolar organizer regions (NOR) variation in fishes of the genus Salvelinus. Copeia 1:47-53.

Phillips RB, Zajicek KD and Utter FM (1986) Chromsome banding in salmonid fishes: nucleolar organizer regions in Oncorhynchus. Can J Genet Cytol 28:502-510.

Porto-Foresti F, Oliveira C, Tabata YA, Rigolino MG and Foresti F (2002) NORs inheritance analysis in crossings including individuals from two stocks of rainbow trout (Oncorhynchus mykiss). Hereditas 136:227-230.

Schmid M, Loser C, Schmidtke J and Engel W (1982) Evolutionary conservation of a common pattern of activity of nucleolous organizer during spermatogenesis in vertebrates. Chromosoma 86:149-179.

Swanson CP, Merz T and Young WJ (1969) Citogenética. EDUSP, São Paulo, 244 pp.

Thorgaard GH (1983) Chromosomal differences among rainbow trout populations. Copeia 3:650-662.

Ueda T and Kobayashi J (1988) Disappearance of Ag-NORs originated from brown trout in the allotriploid female rainbow trout and male brown trout. Proc Japan Acad 63B:51-55.

Zar JH (1984) Biostatistical analysis. 2nd edition. Prentice-Hall, Englewood Cliffs.

Editor: Yatiyo Yonenaga-Yassuda 